\title{
Fat graft survival inside pocket for silicone implant
}

\author{
Fatma Nihal DURMUS KOCAASLAN ${ }^{1}$ (D), Melekber CAVUS OZKAN ${ }^{1}$ (D), Fatma Betul TUNCER ${ }^{2}$ (iD, Mehmet BAYRAMICLI ${ }^{1}$ (iD \\ 1 Department of Plastic Reconstructive and Aesthetic Surgery, School of Medicine, Marmara University, Istanbul, Turkey. \\ 2 Department of Plastic and Reconstructive Surgery, Cleveland Clinic, Ohio, USA. \\ Corresponding Author: Fatma Nihal DURMUS KOCAASLANＥ-mail: dr-nihal@hotmail.com
}

Submitted: 20.01.2020 Accepted: 02.03 .2020

\begin{abstract}
Objective: The aim of this study was to investigate the survival potential of the fat grafts inside the implant capsule in an experimental setting.

Materials and Methods: Twenty male Wistar albino rats were used. A two-staged surgical procedure was performed. In the first stage, silicone sheets were placed in the subcutaneous plane on the left side of each rat. On day 60, fat grafts injected into the silicone capsule either by removing the silicone sheaths or leaving the silicone sheaths in their place. In both groups, the same amount of the fat grafts was injected into the right side of the dorsal subcutaneous plane of the rats and they served as their own controls. The findings were evaluated according to the histopathological criteria.

Results: There was no statistically significant difference in the inflammatory cell infiltration, fibrosis, and necrotic adipocytes among the groups. Although, the fat viability rate was higher in control groups, there was no statistically significant difference compared to the capsule or silicone groups ( $\mathrm{p}>0.05)$.

Conclusion: Our study results suggest that implant capsule is a hospitable environment and resection of the capsule is unnecessary, if silicone implants are expected to be removed following fat injection.

Keywords: Fat graft, Lipofilling, Adipocyte, Capsule, Silicone implant, Prosthesis
\end{abstract}

\section{INTRODUCTION}

The fibrous tissue capsule formation surrounding silicone implants is the natural consequence of inflammatory mechanisms and wound healing processes [1-3]. Silicone implants are widely used in aesthetic and reconstructive breast surgery and are associated with complications, which give rise to many discussions. Despite the debate, an implant capsule is applied as a local flap, a graft material, or even as a vascular carrier which enable the graft to take over itself thanks to its natural, well-vascularized tissue layer [4].

Inrecent years, fat grafting has been increasingly used in aesthetic and reconstructive breast surgery, as it yields symmetry and natural consistency of the breast tissue with the ability of camouflaging the irregularities [5-12]. Tissue layers between the capsule and skin is the favorable plane of injection for the fat grafts [13]. However, there is a relatively limited space for injection without excessive increase in the pressure of the recipient compartment, leading to a higher amount of graft loss. Despite the well-vascularized nature of the implant capsule and a considerably larger space inside the implant pocket, fat injection is practically avoided. In addition, there is a limited number of data regarding the survival of the fat grafts inside the implant pocket, which offers an ample space and well-vascularized bed for fat grafts.

In the present study, we aimed to investigate the survival potential of the fat grafts inside the pocket for the silicone implant in an experimental setting.

\section{MATERIALS and METHODS}

\section{Study population}

All experimental protocols were approved by the Local Ethics Committee (Date: 04.01.2016/No.004.2016.mar) on Animal Experiments at Marmara University, School of Medicine and were under the National Institute of Health guidelines for the Care and the Use of Laboratory Animals. All surgical procedures were performed at the Experimental Animal Laboratory of Marmara University, School of Medicine. 
A total of 20 male Wistar albino rats with a mean weight of 500 (range, 450 to 550) g were used. All subjects were fed with a standard diet and were sedated using ketamine (Ketalar ${ }^{\circledast}$ Pfizer, Turkey) and xylazine (Rompun ${ }^{\oplus}$ Bayer, Turkey) [14,15]. Dorsum of the rats were shaved with an electric razor and prepared for surgery. A non-sterile, but clean surgical technique was used. A two-staged surgical procedure was adopted in all subjects. In the first stage, $2 \times 1-\mathrm{cm}$ silicone sheets were placed in the subcutaneous plane on the left side of each rat through a $1-\mathrm{cm}$ incision (Figure 1). On day 60, the second stage of surgery was performed by harvesting the fat grafts from the inguinal fat pad on both sides of the subjects. The collected specimen was gently minced with scissors and filled into 2 -cc syringes. The implant pockets on both sides of the rat were, then, exposed.

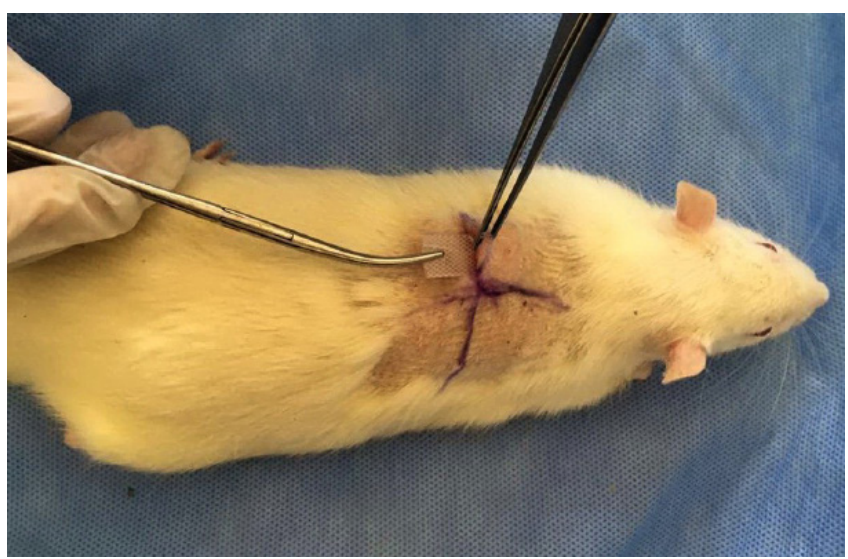

Figure 1. Silicone sheet placement in the subcutaneous plane on left side through a $1-\mathrm{cm}$ incision.

All subjects were divided into four groups according to the presence of the silicone sheath inside the implant pocket. In the first group $(n=10)$, silicone sheaths were removed and $1 \mathrm{cc}$ of the fat graft was injected into the implant pocket (Figure 2). In the second group $(n=10)$, the same amount of the fat graft was injected into the left implant pocket, leaving the silicone sheaths in place. The pockets were, then, closed with three separate absorbable sutures.

In both groups the same volume $(1 \mathrm{cc})$ of the fat graft was implanted into the right side of the dorsal subcutaneous plane of the rats through a separate incision and they served as their own controls. As a result, two study groups were divided into two subgroups as follows:

Group 1 (Left side - Capsule Group) - Fat grafts inside the silicone implant pocket without an implant.

Group 1 Control (Right side - Capsule Control Group) - Subcutaneous fat grafts.

Group 2 (Left side - Silicone Group) - Fat grafts inside the silicone implant pocket with an implant.
Group 2 Control (Right side - Silicone Control Group) - Subcutaneous fat grafts.

All subjects were caged separately and sacrificed at the end of four months. The capsule and fat grafts were identified macroscopically through a midline incision and excised with surgical safety margins. Finally, fat grafts were sent to histological examination in formaldehyde solution.

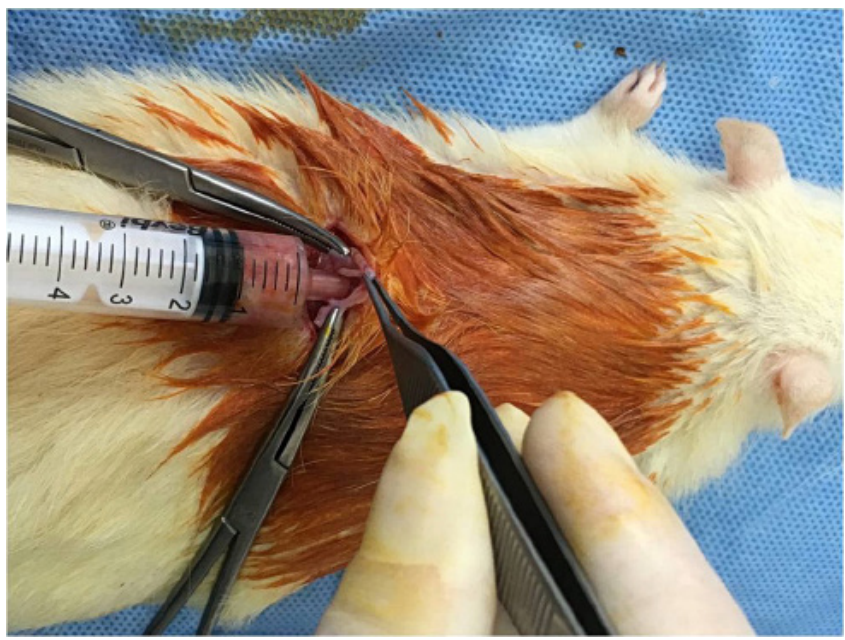

Figure 2. 1-cc of fat graft injection into the implant pocket following silicone sheath removal through previous incision.

\section{Histological examination}

Adipose tissues which were fixed in $10 \%$ formaldehyde solution were dehydrated in ascended alcohol series, clarified in xylene, and embedded in paraffin. A 5- $\mu$ m-thick paraffin sections were stained with hematoxylin \& eosin (H\&E) for histopathological evaluation. In each section, five similar areas were evaluated at x200 magnification through light microscope (Olympus BX-51; Olympus Life Science, Tokyo, Japan) by two blinded histologists and photographed with a digital camera (Olympus DP72, Olympus Corp., Tokyo, Japan). The modified histopathological criteria were inflammatory cell infiltration, presence of cyst formation, fibrosis, fat necrosis, and viable fat tissue [16]. Each criterion was scored as none (0), mild (1), moderate (2), and extensive (3), being a maximum score of 15 .

\section{Statistical Analysis}

Statistical analysis was performed using the Statistical Package for the Social Sciences (SPSS) version 15.0 software (SPSS Inc., Chicago, IL, USA). Descriptive data were expressed in mean \pm standard deviation (SD), median (min-max), or number and frequency. The Kruskal-Wallis test (nonparametric analysis of variance [ANOVA]) was used to analyze significant differences among the groups. A $p$ value of $<0.05$ was considered statistically significant. 


\section{RESULTS}

\section{Macroscopic findings}

Significant vascularity was observed around the capsules filled with fat grafts (Group 1) which had regular borders and a globular shape (Figures 3-5). In the control group, fat grafts were shaped irregularly.

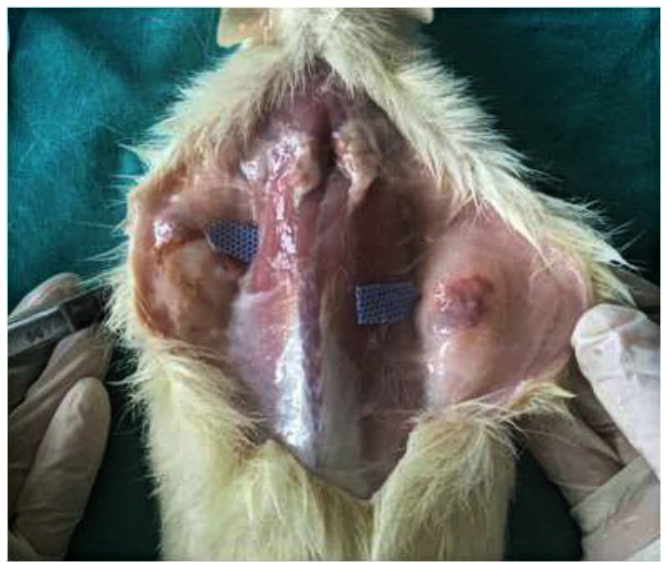

Figure 3. Fat grafts in capsule group on left side and capsule control group on right side.

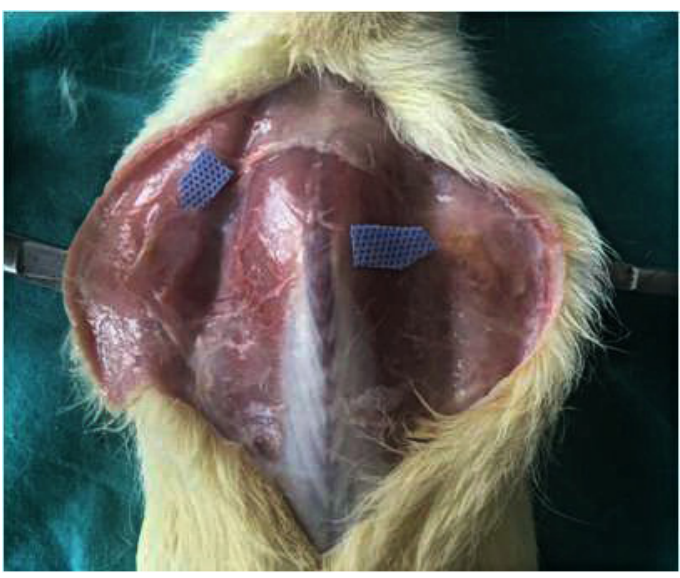

Figure 4. Fat grafts in silicone group on left side and silicone control group on right side.

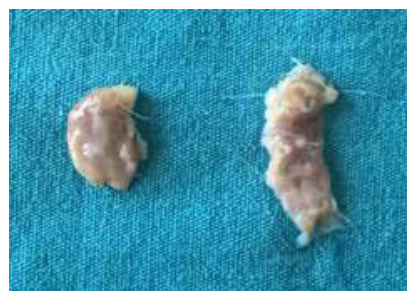

Figure 5. A circular shaped fat graft of capsule group on left side and an irregular-shaped fat graft of capsule control group on right side group are seen.

\section{Microscopic findings}

Histopathological results showed a cyst formation with vascular congestion, inflammatory cell infiltration, mast cells adjacent to the blood vessels, fibrosis, and viable and necrotic adipocytes in both study groups and controls (Figure 6). There was no statistically significant difference in the inflammatory cell infiltration, fibrosis, and necrotic adipocytes among the groups $(0.9931,0.2882,0.8621)$. However, the number of viable adipocytes was higher in the control groups (Table I). Increased degranulated mast cells, vascular congestion, and large cyst formation were moderate in capsule and its control group.

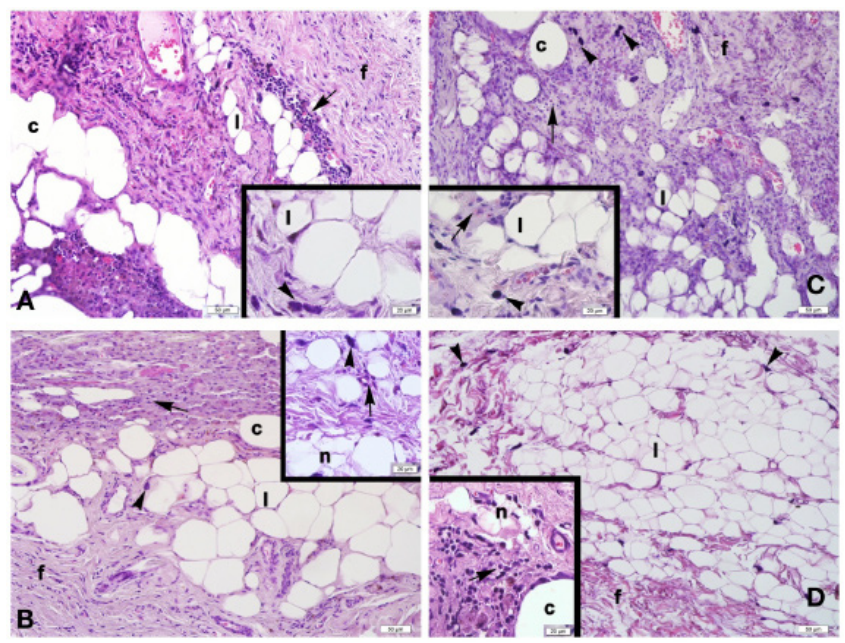

Figure 6. Representative light micrographs seen in experimental groups. Cyst formation (c), inflammatory cell infiltration (arrow), mast cells (arrow head), fibrosis (f), viable (l) and necrotic (n) adipocytes are seen in all groups under H\&E staining (x200); insets (x400. a) Capsule group; b) Capsule control group; c) Silicone group; and d) Silicone control group.

Inflammation scores were similar in all subgroups. Although the fat viability was higher in the control groups, there was no statistically significant difference compared to the capsule or silicone groups $(p=0.2336)$. The cyst formation scores were also similar in both subgroups, indicating no statistically significant difference $(\mathrm{p}=0.2711)$ (Table I). Histopathological results showed cyst formation, vascular congestion, inflammatory cell infiltration, mast cells close to the blood vessels, fibrosis, viable and necrotic adipocytes in all groups. Inflammatory cell infiltration, fibrosis and necrotic adipocytes were similar in all groups. Viable adipocytes were more extant in lipid and silicone control groups. Increased and degranulated mast cells, vascular congestion and large cyst formation were moderate in the lipid experiment group. 
Table I. Modified histopathological criteria scores

\begin{tabular}{|c|c|c|c|c|c|c|}
\hline & Inflammation & Cyst & Fibrosis & Viable fat & $\begin{array}{c}\text { Necrotic } \\
\text { fat }\end{array}$ & Total \\
\hline $\begin{array}{l}\text { Capsule } \\
\text { group }\end{array}$ & $2.25(0.67)$ & $2.8(0.34)$ & $\begin{array}{c}2.15 \\
(0.88)\end{array}$ & $1.9(0.73)$ & $1.4(0.87)$ & $\begin{array}{c}10.5 \\
(1.77)\end{array}$ \\
\hline $\begin{array}{c}\text { Capsule } \\
\text { control } \\
\text { group }\end{array}$ & $2.2(1.13)$ & $2.5(0.97)$ & $2.3(1.15)$ & $2.1(0.84)$ & $1.3(0.94)$ & $\begin{array}{l}10.4 \\
(3.2)\end{array}$ \\
\hline $\begin{array}{l}\text { Silicone } \\
\text { group }\end{array}$ & $2.25(0.75)$ & $\begin{array}{c}2.31 \\
(0.88)\end{array}$ & $\begin{array}{l}1.75 \\
(0.65)\end{array}$ & $\begin{array}{l}1.25 \\
(0.53)\end{array}$ & $1.5(0.92)$ & $\begin{array}{c}9.06 \\
(1.78)\end{array}$ \\
\hline $\begin{array}{c}\text { Silicone } \\
\text { control } \\
\text { group }\end{array}$ & $2.13(0.79)$ & $\begin{array}{c}1.75 \\
(0.88)\end{array}$ & $2.5(0.92)$ & $\begin{array}{c}2.13 \\
(0.95)\end{array}$ & $\begin{array}{l}1.75 \\
(0.88)\end{array}$ & $\begin{array}{l}10.25 \\
(1.90)\end{array}$ \\
\hline$p$ value & $\mathrm{p}=0.9931$ & $\mathrm{p}=0.2711$ & $\mathrm{p}=0.2882$ & $\mathrm{p}=0.2336$ & $\mathrm{p}=0.8621$ & \\
\hline
\end{tabular}

Data are given in mean and standard error (SEM) in brackets, unless otherwise stated.

\section{DISCUSSION}

Fat grafting, which has been performed for more than one hundred years, Coleman [17] first refined the technique in 1997. Since then, autologous fat grafting as a reliable natural filler has been increasingly adopted with more consistent results. Currently, it is used for various purposes in aesthetic and reconstructive breast surgery with most common indications being the aesthetic or reconstructive augmentation of the breast with or without silicone implants, correction deformities and asymmetries of the breast, and the refinement of the soft tissues covering the breast implants $[18,19]$. However, the reabsorption rate of fat grafts widely varies ranging from 0 to $44 \%$ and often undergo fat necrosis [20].

A fibrotic capsule surrounding the silicone implants is the natural result of wound healing processes which aggravates within two months $[1-3,21,22]$. It acts as a barrier between the tissue and the implant. The capsule is a vascular fibrous tissue which receives a significantly higher blood flow than the surrounding soft tissues [23]. Although, it may also serve as an ideal recipient surface for graft harvesting, pericapsular soft tissue is the preferred recipient bed for fat grafts in the clinical practice, and fat injection inside the implant pocket is avoided [13]. On the other hand, data regarding the outcome of the injected fat grafts inside the pocket are scarce. The present study was, therefore, designed to investigate the outcome of fat grafts inside the implant pocket. Our study results showed that fat grafts survived inside the implant pocket in a similar size to the environment following the removal of the implant. Fat survival score of the capsule group was not significantly different than in the control groups. However, this finding is not consistent with the results of Yazawa et al., who reported poor survival of fat grafts inside their pocket for silicone implants without the treatment of basic fibroblast growth factor (bFGF) [24]. In our study, on the other hand, no growth factors or substitutes were available to enhance the vascularity. We used ordinary silicone implants which is compatible with the clinical setting. The discrepancy between the results of Yazawa et al. , and our results can be attributed to the distinct properties of the implanted object in their study. These authors used silicone implants coated with a photoreactive gelatin containing bFGF [24]

It has been well-established that the presence of a foreign body in a physiological environment promotes ongoing stimulation for inflammatory mechanisms which does not offer a welldisposed bed for graft survival $[2,26]$. In our study, fat grafts survived inside the implant pocket even in the presence of implants. However, the survival rate of fat grafts in the silicone group was significantly lower than in the controls. This decline can be explained either with the ongoing foreign body reaction, which increases the resorption rate of the fat grafts, or with the possible pressure effect of the implant. This finding is likely to support the clinical practice which avoids the arrangement of fat grafts inside the implant pockets.

Fibrosis is an indication of fat graft loss due to an unfavorable recipient bed. Another interesting finding of the present study is the lower fibrosis rate in the capsule and silicone groups, compared to relevant control groups. Although, increased neutrophil and fibroblast infiltration into the implant capsule has already been reported in the literature [1,27] , extensive collagen deposition might have isolated the fat grafts from the surrounding tissues as a barrier to reduce the fibroblast migration into the grafts.

The geometric effect of the implant capsule on fat graft survival is another major theme. Fat grafts in the capsule group had welldefined borders with a spherical shape. The silicone capsule served as a three-dimensional scaffold for the fat grafts and might have provided surgeons with another reconstructive option with fat grafts to achieve the desired shape and size.

Nonetheless, there are some limitations to this study. The low sample size might have contributed to the bias. In our experimental design, each subject served as its own control to reduce the amount of error in a limited sample size. Geometry of the implant pockets can be considered another limitation. Fibrous capsule pockets in our study were formed by the implantation of flat silicone sheaths offering a better contact surface for the fat grafts to survive. However, in the relevant clinical scenario, the implant pockets would be more extensive and rounder. Therefore, the viability of graft lobules at the core of the injection bulk would be questionable.

\section{Conclusion}

In conclusion, to the best of our knowledge, this is the first controlled study to investigate the outcome of the fat grafts inside the silicone implant pockets without using any intervention for vascularity enhancement. Our study results indicate that a pocket 
for the silicone implant is a hospitable environment for fat grafts following the removal of the implant. In addition, resection of the capsule seems to be unnecessary, if silicone implants are expected to be removed following fat injection. Although, our results in the experimental setting demonstrate that the survival rate of fat grafts inside the implant pocket confirms a satisfactory three-dimensional shape, further large-scale studies with higher volumes of fat grafting in larger pockets are required to establish its clinical relevance.

\section{Compliance with Ethical Standards}

Funding: The study was not supported by any funds.

Conflict of interest: The authors have no conflicts of interest to declare.

Ethical approval: The study protocol was approved by the Local Ethics Committee (Date: 04.01.2016/No.004.2016. mar) on Animal Experiments at Marmara University, School of Medicine and were under the National Institute of Health guidelines for the Care and the Use of Laboratory Animals. All surgical procedures were performed at the Experimental Animal Laboratory of Marmara University, School of Medicine.

\section{REFERENCES}

[1] Silva EN, Ribas-Filho JM, Czeczko NG, et al. Histological evaluation of capsules formed by silicone implants coated with polyurethane foam and with a textured surface in rats. Acta Cir Bras 2016 ;31:774-82. doi: 10.1590/ S0102.865.0201601.200.00001

[2] Imber G, Schwager RG, Guthrie RH Jr, Gray GF. Fibrous capsule formation after subcutaneous implanation of synthetic materials in experimental animals. Plast Reconstr Surg 1974 ;54:183-6.

[3] Wagner H, Beller FK, Pfautsch M. Electron and light microscopy examination of capsules around breast implants. Plast Reconstr Surg 1977;60:49-55.

[4] Sarifakioglu N, Gökrem S, Bingul F, Ustun H, Aslan G. The fate of transplanted fibrous capsule as an autogenous graft. Plast Reconstr Surg 2005 ; 115:1087-94.

[5] Simonacci F, Grieco MP, Bertozzi N, Raposio E. Autologous fat transplantation for secondary breast reconstruction: our experience. G Chir. 2017 May-Jun;38(3):117-123.

[6] Streit LM D, Lhotsky R, Mestak O. Autologous fat transfer, breast lipomodelling and fat transfer to the face: Current gold standards and emerging new data. Acta Chir Plast 2017 ;59:97-108.

[7] Chiu CH. Does Stromal Autologous fat transfer, breast lipomodelling and fat transfer to the face: Current gold standards and emerging new data. Aesthet Surg J 2018 Feb 9. [Epub ahead of print]

[8] Boccara D, Haddad J, Chaouat M, Serror K, Mimoun M. Treating breast conservation therapy defects with brava and fat grafting: technique, outcomes, and safety profile. Plast Reconstr Surg 2018;141:965e. doi: 10.1097/PRS.000.000.0000004299.
[9] Delay E, Meruta AC, Guerid S. Indications and controversies in total breast reconstruction with lipomodeling. Clin Plast Surg2018;45:111-7. doi: 10.1016/j.cps.2017.08.009.

[10] Delay E, Guerid S, Meruta AC. Indications and controversies in lipofilling for partial rreast reconstruction. Clin Plast Surg2018;45:101-10. doi: 10.1016/j.cps.2017.08.008.

[11] Delay E, Garson S, Tousson G, Sinna R. Fat injection to the breast: technique, results, and indications based on 880 procedures over 10 years. Aesthet Surg J 2009 ;29:360-76. doi: 10.1016/j.asj.2009.08.010.

[12] Spear SL, Wilson HB, Lockwood MD. Fat injection to correct contour deformities in the reconstructed breast. Plast Reconstr Surg 2005;116:1300e5.

[13] Hammond DC, O'Connor EA, Scheer JR. Total envelope fat grafting: a novelapproach in breast reconstruction. Plast Reconstr Surg 2015;135:691-4. doi: 10.1097/PRS.000.000.0000000968.

[14] FishRE. Anesthesia and Analgesia in Laboratory Animals. 2nd ed. ACLAM, London:Academic Press, 2008.

[15] Bayramiçli M.Anaesthesia, analgesia and drugs. In Bayramiçli M, ed. Experimental Microsurgery-Basic Research, Tissue and Organ Transplantation Models (Turkish). Istanbul:Argos Publications, 2005: 207-30.

[16] Condé-Green A, Wu I, Graham I, et al. Comparison of 3 techniques of fat grafting and cell-supplemented lipotransfer in athymic rats: a pilot study. Anesthet Surg J 2013 ;33:713-21. doi: $10.1177 / 1090820 X 13487371$.

[17] Coleman SR. Facial recontouring with lipostructure. Clin Plast Surg 1997 ;24:347-67.

[18] [18]. Simonacci F, Bertozzi N, Grieco MP, Grignaffini E, Raposio E. Autologous fat transplantation for breast reconstruction: A literature review. Ann Med Surg (Lond) 2016 ;12:94-100. eCollection 2016 Dec.

[19] Coleman SR, Saboeiro AP. Fat grafting to the breast revisited: safety and efficacy, Plast Reconstr Surg2007;119:775-85.

[20] Beck M, Amar O, Bodin F, Lutz JC, Lehmann S, Bruant-Rodier C. Evaluation of breast lipofilling after sequelae of conservative treatment for cancer. EurJPlastic Surg 2012 ;35:221-8. doi: 10.1007/s00238.011.0606-X

[21] Taylor SR. Effect of surface texture on the soft tissue response to polymer implants. J Biomed Mater Res1983;17:205-27.

[22] Thomson H. The fate of the pseudosheath pocket around silicone implants. Plast Reconstr Surg 1973 ;51:667-71.

[23] Imran D, Javaid M, Lewis D, Attar KH. Capsular flap for correction of contour deformities of the breast. Ann Plast Surg 2005;54:662-3.

[24] Yazawa M, Mori T, Tuchiya K, Nakayama Y, Ogata H, Nakajima T. Influence of vascularized transplant bed on fat grafting. Wound Rep Reg 2006 ;14:586-92.

[25] Yager DR, Nwomeh BC. The proteolytic environment of chronic wounds. Wound Repair Regen 1999 ;7:433-41.

[26] Majd H, Scherer BSS, Boo S, et al. Novel micropatterns mechanically control fibrotic reactions at the surface of silicone implants. Biomaterials $2015 ; 54: 136-47$. doi: 10.1016/j. biomaterials.2015.03.027. 
[27] Wagenführ-Júnior J, Ribas Filho JM, Nascimento MM, Ribas FM, Wanka MV, Godoi Ade L. Histopathological reaction over prosthesis surface covered with silicone and polyurethane foam implanted in rats. Acta Cir Bras 2012 ;27:866-73. 